\title{
Clinical Characteristics and Outcome of COVID-19 among People Living with Diabetes in Nigeria
}

\author{
Vivian Gga Kwaghe ${ }^{1 *}$, Rifkatu Reng ${ }^{2}$, Olufemi Adediran ${ }^{2}$ and Felicia Anumah ${ }^{2}$ \\ ${ }^{1}$ Infectious Disease Unit, Department of Internal Medicine, University of Abuja Teaching Hospital, \\ Gwagwalada, Abuja, Nigeria \\ ${ }^{2}$ Endocrinology Unit, Department of Internal Medicine, University of Abuja Teaching Hospital, \\ Gwagwalada, Abuja, Nigeria
}

*Corresponding author: Vivian Gga Kwaghe, Infectious Disease Unit, Department of Internal Medicine, University of Abuja Teaching Hospital, Gwagwalada, Abuja, Nigeria

\begin{abstract}
Introduction: The novel coronavirus disease 2019 (COVID-19) has affected millions of people globally with over 3 million deaths. People with underlying comorbidities are at a risk of having severe disease with increased fatality. This study aimed at describing the clinical characteristics and outcome of COVID-19 among people living with diabetes mellitus (DM) in Nigeria.

Methodology: This was a retrospective study that analyzed the data of adult patients who were admitted into the isolation and treatment center at the University of Abuja Teaching Hospital (UATH) in Nigeria, with a diagnosis of COVID-19 from March 2020 to March 2021. Data on demographic and clinical features as well as outcome was extracted and analyzed using the Statistical Package for Social Sciences (SPSS) version 20.0 .

Results: A total of 588 adult patients were hospitalized with COVID-19 during the study period; $364(61.9 \%)$ of who were males with a male: female ratio of $1.81: 1$. The commonest symptoms at presentation were fever (56.3\%), cough $(54.3 \%)$, shortness of breath $(37.6 \%)$, headache $(14.5 \%)$, sore throat $(9.2 \%)$, anosmia $(6.5 \%)$, rhinorrhea $(5.6 \%)$ and diarrhea (5.3\%). Of the 588 patients, 169 $(28.7 \%)$ had diabetes; $109(64.5 \%)$ of whom were males. Patients with DM were older than those without DM $(p<$ 0.0001 ), and had a high proportion of symptoms of fever, cough and shortness of breath $(p=0.001)$. They had more severe disease $(p=0.0001)$ with a higher mortality rate $(22.5 \%)$ when compared to those without DM (12.5\%) (p $=0.003)$.
\end{abstract}

Conclusion: DM is among the most frequently reported comorbidities in patients infected with COVID-19 and is associated with a higher risk of severe and fatal forms of the disease. People living with DM in Nigeria are 1.8 times more likely to die from COVID-19 than people who do not have DM.

\section{Keywords}

COVID-19, Diabetes, Clinical characteristics, Outcome, Nigeria

\begin{abstract}
Abbreviations
COVID-19: Coronavirus Disease 2019; UATH: University of Abuja Teaching Hospital; WHO: World Health Organization; SARS-CoV-2: Severe Acute Respiratory Syndrome Coronavirus 2; DM: Diabetes Mellitus; FCT: Federal Capital Territory; RNA: Ribonucleic Acid; IFN: Interferon; SBP: Systolic Blood Pressure; DBP: Diastolic Blood Pressure; SPO2: Oxygen Saturation; WBC: White Blood Count; RBS: Random Blood Sugar; CRP: C-Reactive Protein; LDH: Lactate Dehydrogenase
\end{abstract}

\section{Introduction}

In December 2019, a new beta coronavirus causing pneumonia emerged in Wuhan, Hubei Province of China. This virus was later identified and called Severe Acute Respiratory Syndrome Coronavirus 2 (SARS-CoV-2) [1]. The disease was given the name Coronavirus Disease 2019 (COVID-19) by the World Health Organization (WHO) in February 2020. It rapidly spread to the rest of the World and was declared a global pandemic by the WHO on the $11^{\text {th }}$ of March 2020 [2]. As of $22^{\text {nd }}$ June 2021 , there have been confirmed $178,360,849$ cases of

Citation: Kwaghe VG, Reng R, Adediran O, Anumah F (2021) Clinical Characteristics and Outcome of COVID-19 among People Living with Diabetes in Nigeria. Int J Diabetes Clin Res 8:147. doi. org/10.23937/2377-3634/1410147

Accepted: August 26, 2021: Published: August 28, 2021

Copyright: (C) 2021 Kwaghe VG, et al. This is an open-access article distributed under the terms of the Creative Commons Attribution License, which permits unrestricted use, distribution, and reproduction in any medium, provided the original author and source are credited. 
COVID-19, including $3,869,384$ deaths, reported to the WHO [3].

Coronaviruses are single stranded RNA viruses, named for the crown-like spikes on their surface. There are four main sub-groupings of coronaviruses, known as alpha, beta, gamma, and delta [4]. Human coronaviruses were first identified in the mid-1960s. The seven coronaviruses that can infect man are: $229 \mathrm{E}$ (alpha coronavirus), NL63 (alpha coronavirus), OC43 (beta coronavirus), HKU1 (beta coronavirus), MERS-CoV (beta coronavirus), SARS-CoV (the beta coronavirus), and SARS-CoV-2 (the novel beta coronavirus that causes COVID-19).

Traditionally, coronaviruses cause a mild respiratory illness. However, it has become apparent that coronaviruses can also cause severe disease; first the SARS-CoV caused an outbreak of respiratory disease originally in China in 2003 and MERS-CoV caused an outbreak in Saudi Arabia in 2012. Since the outbreak of COVID-19, emerging data has shown that people with underlying comorbidities like diabetes mellitus, hypertension, chronic kidney disease, Human Immunodeficiency Virus, chronic obstructive pulmonary disease as well as the elderly are at a risk of developing severe disease with a higher mortality rate $[5,6]$.

Diabetes mellitus (DM) is a metabolic disorder of chronic hyperglycemia characterized by disturbances to carbohydrate, protein, and fat metabolism resulting from absolute or relative insulin deficiency with dysfunction in organ systems [7]. Data published by the International Diabetes Federation (IDF) in 2017 showed that 425 million persons were living with DM worldwide, with nearly $50 \%$ of these undiagnosed [8]. The prevalence of DM in Nigeria is estimated at $5.77 \%$; with a population of about 200 million, this suggests that about 11.5 million Nigerians are living with the disease [9].

Early in the pandemic, DM emerged as a medical condition that is associated with severe COVID-19. Many pathophysiological abnormalities occur in patients with DM that make them more susceptible to infections. Patients with DM have a deficiency of complement C4 protein [10]. The complement proteins promote the opsonization and phagocytosis of microorganisms through macrophages and neutrophils and induce the lysis of these microorganisms. A deficiency of a complement protein will ultimately lead to a defect in the opsonization and phagocytosis of microorganisms. Secondly, the hyperglycemic environment in DM increases apoptosis of polymorphonuclear leukocytes, and reduces their ability to transmigrate through the endothelium [11]. Also, there is dysregulation of nitric oxide (NO) production in response to bradykinin leading to vasoconstriction, which could attenuate the ability of phagocytes to reach their targets [12]. Thirdly, glycation of immunoglobulins also occur in patients with $D M$, leading to a decrease in the function of the immunoglobulins [13]. Fourthly, it has also been shown that patients with DM have a decreased antiviral response demonstrated by a diminished production of IFN- $\alpha$ and IFN-1 [14]. These processes lead to a defective immune function in patients with DM, making them susceptible to bacterial and viral infections.

Angiotensin converting enzyme-2 (ACE-2) is a plasma membrane protein expressed largely in the lungs as well as in many other tissues and endothelial cells including the heart, kidneys, and importantly in insulin producing $\beta$-cells. SARS-CoV-2 virus has a high affinity towards ACE2 which acts as the "receptor" for SARS-CoV-2 and is the dominant pathway through which the virus gains entry into the lungs and other cells in the body $[15,16]$. Using a Mendelian randomization analyses, Rao, et al. identified the association between diabetes and higher lung ACE2 expression which may predispose DM patients to increase susceptibility to SARS-CoV-2 infection [17]. Also, the use of ACE-2 inhibitors and Angiotensin Receptor Blockers (ARBs) in the treatment of DM and hypertension can up regulate the expression of the ACE- 2 receptors as an adaptive response to counteract the elevated levels of angiotensin-II, thereby leading to increased susceptibility to SARS-CoV-2 infection [18]. Furthermore, Furin, a type 1 membrane-bound protease expressed in high levels in DM patients activates the spike protein $S$ on the SARS-CoV- 2 to bind to the ACE2 receptors; thereby increasing their susceptibility to COVID-19 [19]. It is therefore not surprising that people living with DM may have an increased susceptibility to COVID-19.

Nigeria was not left out of the pandemic as the Country recorded the first case of COVID-19 on the $27^{\text {th }}$ of February 2020. So far over 167 thousand people have been infected with over two thousand deaths. With a large number of the population living with DM it becomes imperative that we understand how COVID-19 affects them. This study therefore aimed at describing the clinical characteristics and outcome of COVID-19 among people living with DM in Nigeria.

\section{Methodology}

\section{Study site}

The study was conducted at the University of Abuja Teaching Hospital (UATH), which is located in the capital city of Abuja, Nigeria. The hospital is a 520 bedded tertiary healthcare facility that was assigned the responsibility for the treatment of moderate to severe cases of COVID-19 patients by the Federal Ministry of Health.

\section{Study design}

The study was a single-centered retrospective study that analyzed the data of adult patients who were admitted into the isolation and treatment center at 
UATH with a diagnosis of COVID-19 from March 2020 to March 2021. The diagnosis of COVID-19 was done by real-time polymerase chain reaction (RT-PCR) using nasopharyngeal and oropharyngeal swabs according to the Nigerian Center for Disease Control guideline. A patient was defined as having moderate COVID-19 if they were breathless but oxygen saturation at room air was greater than 90\%; while severe COVID-19 was defined as breathlessness plus oxygen saturation at room air less than $90 \%$. Diabetes was defined as self-reported medical history of diabetes, and the use of anti-diabetic drugs. We reviewed the clinical records of the patients and extracted data on demographic characteristics, clinical symptoms, underlying comorbidities, essential examination findings including pulse rate, blood pressure on admission, oxygen saturation (at room air), investigation findings and clinical outcomes. The primary outcome of interest was either discharged or death.

\section{Data analysis}

Data was analyzed using the Statistical Package for Social Sciences (SPSS) version 20. Continuous variables were expressed as mean and standard deviation. Categorical variables were analyzed as counts and percentages. Chi-square analysis was used to determine the significance of difference between categorical variables. A value of $p<0.05$ was considered statistically significant.

\section{Ethical approval}

Data used for this study was based on secondary data analysis and ethical approval is not required. The UATH hospital management gave the approval for the release and use of the data which was de-identified to ensure anonymity.

\section{Results}

\section{Clinical features of the general population}

The baseline characteristics of the study subjects are provided in Table 1 and Table 2. A total of 588 adult patients were hospitalized with COVID-19 during the study period; $364(61.9 \%)$ of who were males with a male:female ratio of 1.81:1. The age range was from 18 years to 103 years with a mean age of 51 ( \pm 16.8 ) years. The commonest symptoms at presentation were fever (56.3\%), cough (54.3\%), shortness of breath (37.6\%), headache $(14.5 \%)$, sore throat $(9.2 \%)$, anosmia $(6.5 \%)$, rhinorrhea (5.6\%) and diarrhea (5.3\%). Other symptoms such as abdominal pain (1.9\%) and myalgia (1.7\%) were less common. Based on the definition of disease

Table 1: Demographic and clinical symptoms.

\begin{tabular}{|c|c|c|c|c|}
\hline & Overall (\%) & DM (\%) & NO DM (\%) & $P$ value \\
\hline Mean Age (years) & $51 \pm 16.8$ & $59.7 \pm 11.1$ & $47.4 \pm 17.3$ & 0.0001 \\
\hline \multicolumn{5}{|l|}{ Gender } \\
\hline Female & $223(37.9)$ & $60(35.5)$ & $163(38.9)$ & 0.37 \\
\hline Male & $365(62.1)$ & $109(64.5)$ & $256(61.1)$ & \\
\hline \multicolumn{5}{|l|}{ Clinical Features } \\
\hline Fever & $331(56.3)$ & $115(68.0)$ & $216(51.6)$ & 0.0001 \\
\hline Cough & $319(54.3)$ & $115(68.0)$ & $204(48.7)$ & 0.0001 \\
\hline Shortness of breath & $221(37.6)$ & $84(49.7)$ & $137(32.7)$ & 0.0001 \\
\hline Headache & $85(14.5)$ & $27(16.0)$ & $58(13.8)$ & 0.477 \\
\hline Sore throat & $54(9.2)$ & $17(10.1)$ & $37(8.8)$ & 0.616 \\
\hline Vomiting & $44(7.5)$ & $16(9.5)$ & $28(6.7)$ & 0.233 \\
\hline Anosmia & $38(6.5)$ & $9(5.3)$ & $29(6.9)$ & 0.492 \\
\hline Diarrhea & $31(5.3)$ & $13(7.7)$ & $18(4.3)$ & 0.09 \\
\hline Loss of appetite & $24(4.1)$ & $9(5.3)$ & $15(3.6)$ & 0.321 \\
\hline
\end{tabular}

Table 2: Vital signs and laboratory parameters.

\begin{tabular}{|c|c|c|c|c|}
\hline \multirow[b]{2}{*}{ vital signs/Lab parameter } & \multicolumn{3}{|l|}{ Diabetes } & \multirow[b]{2}{*}{$P$ Value } \\
\hline & Total & Yes & No & \\
\hline SPO2 (\%) & $93.93 \pm 9.92$ & $89.79 \pm 12.44$ & $94.85 \pm 8.28$ & $<0.0001$ \\
\hline TEMP $\left({ }^{\circ} \mathrm{C}\right)$ & $36.51 \pm 0.59$ & $36.5 \pm 0.5$ & $36.52 \pm 0.63$ & 0.7193 \\
\hline Heart rate (b/min) & $93.67 \pm 16.99$ & $95.72 \pm 17.97$ & $92.84 \pm 16.52$ & 0.0638 \\
\hline $\mathrm{SBP}(\mathrm{mmHg})$ & $133.33 \pm 21.38$ & $136.55 \pm 23.09$ & $132 \pm 20.52$ & 0.0203 \\
\hline $\mathrm{DBP}(\mathrm{mmHg})$ & $84.28 \pm 14.11$ & $84.4 \pm 14.46$ & $84.22 \pm 13.98$ & 0.888 \\
\hline WBC (10\%/L) & $10.44 \pm 8.25$ & $11.08 \pm 8.66$ & $10.17 \pm 8.07$ & 0.2708 \\
\hline RBS (mmol/L) & $9.22 \pm 5.46$ & $9.54 \pm 5.28$ & $9.08 \pm 5.55$ & 0.5364 \\
\hline
\end{tabular}


severity, 103 (17.5\%) patients had severe disease while 485 (82.5\%) patients had mild to moderate disease. Disease severity was higher among those with DM (31.4\%) compared to those without DM (11.9\%) (P < 0.0001). A total of 140 (23.8\%) patients had underlying comorbidities; the commonest being hypertension (51.4\%), diabetes (28.7\%), asthma (3.4\%) and HIV (3.4\%).

\section{COVID-19 in people living with DM}

Out of the 588 patients hospitalized with COVID-19 during the study period, 169 (28.7\%) had diabetes; 109 $(64.5 \%)$ of whom were males. The age range of those with DM was 27-88 years, with a mean age of 59.7 ( \pm 11.1 ) years. The patients with DM were older (mean age 59.7 years) compared to the patients without DM who had a mean age of $47.4( \pm 17.3)$ years $(p<0.0001)$. The clinical symptoms were the same for both the DM and the non DM patients. However, patients with DM had more symptoms of Fever (68\% versus 51.6\%), cough (68\% versus $48.7 \%)$ and shortness of breath $(49.7 \%$ versus $32.7 \%$ ) when compared to patients without DM $(p<0.001)$.

Patients with DM presented with lower oxygen saturation $(89.7 \pm 12.4)$ compared to those without DM (94.85 \pm 8.28$)(p<0.0001)$. The mean systolic blood pressure was higher among the DM group compared to the non DM group (136.55 $\mathrm{mmHg}$ versus $132.0 \mathrm{mmHg}$ ) $(p=0.02)$. There was no difference in the mean diastolic blood pressure, temperature and mean random blood sugar at presentation between the DM patients and the non DM patients (Table 2).

\section{Outcome}

The primary outcome of interest was either discharged or death (Table 3). A total of 497 (84.5\%) patients were successfully treated and discharged home in a stable condition; while 91 (15.5\%) patients died. Out of the 169 patients who had DM, 38 (22.5\%) died. Mortality rate was higher among the DM group (22.5\%) when compared to the mortality rate among those without DM (12.6\%) ( $p=0.003)$. The patients spent between 0 days to 43 days on hospitalization; the average length of hospital stay was $10( \pm 5.7)$ days. Those with DM had a mean hospital stay of $9.3( \pm 5)$ days; while those without DM had a mean hospital stay of $10.3( \pm 5.9)$ days. The difference was however not statistically significant $(p=0.104)$.

\section{Discussion}

Diabetes is among the most frequently reported comorbidities in patients infected with COVID-19 [20-22]. In this retrospective study of 588 patients hospitalized with COVID-19 at UATH, 28.7\% were patients living with DM. This is about 5 times the prevalence of DM in the general population in Nigeria which is estimated at $5.77 \%$. Orioli, et al. reporting from Belgium found a prevalence of $21.1 \%$, which was 3 -fold higher than the prevalence of DM in the general adult population [23]. Yan, et al. in a similar study in China reported that $24.9 \%$ of the patients with severe COVID-19 had diabetes; the prevalence of DM in the Chinese population has been estimated at $11.6 \%$ [24]. These epidemiologic evidences show that people living with DM are not only susceptible to bacterial infections but also to SARS-CoV-2.

This study showed that patients with DM were older when compared to those without DM $(p=0.0001)$. Older adults are at high risk for the development of type 2 DM due to the combined effects of increasing insulin resistance and impaired pancreatic islet function with aging. A decline in lean body mass and an increase in body fat that often accompanies aging may contribute to the development of insulin resistance [25]. Agerelated insulin resistance is associated with physical inactivity in the elderly. Zhou, et al. from China in a similar study also showed that COVID-19 patients with DM were older than those without DM [26]. With improvement in healthcare in most parts of the World, people are living longer and this unprecedented aging of the world's population is a major contributor to the diabetes epidemic [27].

We also observed that patients with DM had a similar pattern of clinical characteristics of COVID-19 compared to patients without DM. Patients with DM however had a high proportion of symptoms of fever (68\% versus $51.6 \%)$, cough (68\% versus $48.7 \%)$ and shortness of breath $(49.7 \%$ versus $32.7 \%)(p=0.001)$. Yan, et al. reporting from China also showed that the clinical characteristics of COVID-19 were similar in both the patients with DM and those without DM [24]. Disease severity was higher among the patients with

Table 3: Disease severity and outcome.

\begin{tabular}{|c|c|c|c|c|}
\hline & Overall (\%) & DM (\%) & No DM (\%) & P-Value \\
\hline \multicolumn{5}{|l|}{ Disease Severity } \\
\hline Severe & $103(17.5)$ & $53(31.4)$ & $50(11.9)$ & 0.0001 \\
\hline Mild-Moderate & $485(82.5)$ & $116(68.6)$ & $369(88.1)$ & \\
\hline \multicolumn{5}{|l|}{ Outcome } \\
\hline Dead & $91(15.5)$ & $38(22.5)$ & $53(12.6)$ & 0.003 \\
\hline Discharged & $497(84.5)$ & $128(75.7)$ & $369(88.1)$ & \\
\hline Length Of Hospital Stay (Days) & $10 \pm 5$ & $9.3 \pm 5$ & $10.3 \pm 5.9$ & 0.104 \\
\hline
\end{tabular}


DM (31.4\%) compared to those without DM (11.9\%) $(p<0.001)$. Earlier reports from China, US and Italy showed that COVID-19 patients with DM often have severe disease with poor outcome [28-30]. A metaanalysis by Kumar, et al. showed that Diabetes was associated with severe COVID-19 with a pooled odds ratio of 2.75 (95\% Cl: 2.09-3.62) [31]. de Almeida-Pititto, et al. in another meta-analysis also identified a 2.3 -fold increase in the risk of severity and a 2.5 -fold increase for mortality associated with COVID-19 in patients with DM [32]. Diabetes is associated with a low grade chronic inflammation induced by the excessive visceral adipose tissue; C-reactive protein (CRP), IL-1 $\beta$, IL- 6 and other cytokines are elevated in patients with DM [33]. When a person living with DM gets infected with SARSCoV-2, the pre-existing chronic inflammation is further increased and plays a critical role in disease progression. Hyperglycemia also increases the formation of advanced glycation end products (AGEs) that stimulate the production of reactive oxygen species, leading to increased oxidative stress and activation of immune response [34]. These processes lead to severe COVID-19 with a poor outcome in people living with DM. Guo, et al. reported that DM patients with COVID-19 had higher levels of C-reactive protein (32.8 [IQR, 11.3-93] vs. 16.3 [IQR, 7.17-43.9]), Erythrocyte sedimentation rate (67 [47.5-81] vs. 23 [10-49]), as well as D-dimer (1.15 [IQR, $0.83-2.11$ ] vs. 0.54 [0.25-1.1]) [35]. In another report by Zhu, et al. they observed that DM patients with COVID-19 had greater incidences of decreased lymphocyte counts and increased neutrophil counts, as well as higher levels of serum interleukin-6 (IL-6), C-reactive protein (CRP), and lactate dehydrogenase (LDH) [36]. These reports show that patients with diabetes are at higher risk of excessive uncontrolled inflammatory responses and hypercoagulable state, which may contribute to a poorer prognosis of COVID-19. In addition, people living with DM are characterized by pulmonary dysfunction due to decreased lung volume, reduced pulmonary diffusing capacity, as well as ventilation control, bronchomotor tone and noradrenergic innervation impairment [37]. These pulmonary changes contribute to a poor outcome when DM patients get infected with SARS-CoV-2.

The mortality rate in this study was $22.5 \%$ among the patients with DM as compared to $12.6 \%$ in the non DM group. This difference was statistically significant $(P=$ 0.003). Patients with DM were 1.8 times more likely to die from COVID-19. Acharya, et al. reported a mortality rate of $20.0 \%$ among COVID-19 patients with DM in Korea; which was 4.2 times higher than the mortality rate among non DM patients with COVID-19 [38]. In a meta-analysis by Corona, et al. that studied 87 articles with 35,486 patients, mortality was higher in COVID-19 patients with DM in the USA and in Europe [39]. Zhu, et al. found a mortality rate of $7.8 \%$ versus $2.7 \%$ ( $p<$ 0.001 ) for DM and non-DM patients respectively, with COVID-19 in a similar study in China [36]. They went further to categorize the DM patients into those with well controlled blood glucose and those with poor glycemic controlled and observed that those with wellcontrolled blood glucose had higher lymphocyte counts, lower neutrophil counts, and lower serum levels of IL-6, CRP, and LDH. They further noticed that the in-hospital death rate was significantly lower $(1.1 \%$ versus $11.0 \%)$ in the well-controlled group relative to the poorly controlled group. This report shows the benefit of a good blood glucose control in the management of DM patients with COVID-19.

\section{Conclusion}

Though infection by SARS-CoV-2 causes mild disease in a majority of people, severe disease is seen in people with underlying comorbidities. DM is among the most frequently reported comorbidities in patients infected with COVID-19. This study showed that $28.7 \%$ of COVID-19 patients hospitalized in a tertiary hospital in Nigeria were people living with DM. The study also showed that DM was associated with a higher risk of severe and fatal forms of COVID-19. People living with DM were 1.8 times more likely to die from COVID-19 than people who do not have DM. More intensive attention should be paid to DM patients with COVID-19 in order to prevent a poor outcome.

\section{Limitations}

This study was a single-centered retrospective study. Diabetes was defined as self-reported medical history of diabetes; some patients with DM who were unaware of their status before they became infected may have been missed. Also, information on the prehospitalization glycemic control was not available.

\section{Competing Interests}

The authors declare no competing interest.

\section{Acknowledgements}

We acknowledge the Chief Medical Director of UATH for his support and the management of UATH for their consent to release the data for this study.

\section{Conflict of Interest}

The authors declare that there is no conflict of interest.

\section{References}

1. Gorbalenya AE, Baker SC, Baric RS, de Groot RJ, Drosten C, et al. (2020) Severe acute respiratory syndrome-related coronavirus: The species and its viruses - a statement of the Coronavirus Study Group. Nature Microbiology 5: 536544.

2. Cucinotta D, Vanelli M (2020) WHO declares COVID-19 a pandemic. Acta Biomed 91: 157-160.

3. https://covid19.who.int/

4. https://www.cdc.gov/coronavirus/types.html 
5. Hasan E, Abdullah A, Aizza Z, Humera J, Kashaf J, et al (2020) COVID-19 and comorbidities: Deleterious impact on infected patients. J Infect Public Health 13: 1833-1839.

6. Zhang J, Wang X, Jia X, Li J, Hu K, et al. (2020) Risk factors for disease severity, unimprovement, and mortality of COVID-19 patients in Wuhan, China. Clin Microbiol Infect 26: 767-772.

7. WHO (1999) Definition, diagnosis and classification of diabetes mellitus and its complications. Part 1: Diagnosis and classification of diabetes mellitus. WHO, Geneva, Switzerland.

8. International Diabetes Federation (2017) Diabetes atlas. (8th edn), International Diabetes Federation, Brussels, Belgium.

9. Uloko AE, Musa BM, Ramalan MA, Gezawa ID, Puepet $\mathrm{FH}$, et al. (2018) Prevalence and risk factors for diabetes mellitus in Nigeria: A systematic review and meta-analysis. Diabetes Ther 9: 1307-1316.

10. Flyvbjerg A (2010) Diabetic angiopathy, the complement system and the tumor necrosis factor superfamily. Nat Rev Endocrinol 6: 94-101.

11. Nirmal J, Caputo GM, Weitekamp MR, Karchmer AW (1999) Infections in patients with diabetes mellitus. N Engl J Med 341: 1906-1912.

12. Santilli F, Cipollone F, Mezzetti A, Chiarelli F (2004) The role of nitric oxide in the development of diabetic angiopathy. Horm Metab Res 36: 319-335.

13. Peleg AY, Weerarathna T, McCarthy JS, Davis TM (2007) Common infections in diabetes: Pathogenesis, management and relationship to glycaemic control. Diabetes Metab Res Rev 23: 3-13.

14. Summers KL, Marleau AM, Mahon JL, McManus R, Hramiak I, et al. (2006) Reduced IFN-alpha secretion by blood dendritic cells in human diabetes. Clin Immunol 121: 81-89.

15. Wu YC, Chen CS, Chan YJ (2020) The outbreak of COVID-19: An overview. J Chin Med Assoc 83: 217-220.

16. Rothan HA, Byrareddy SN (2020) The epidemiology and pathogenesis of coronavirus disease (COVID-19) outbreak. J Autoimmun 109: 102433.

17. Rao S, Lau A, So HC (2020) Exploring diseases/traits and blood proteins causally related to expression of ACE2, the putative receptor of SARS-CoV-2: A mendelian randomization analysis highlights tentative relevance of diabetes-related traits. Diabetes Care 43: 1416-1426.

18. Fang L, Karakiulakis G, Roth M (2020) Are patients with hypertension and diabetes mellitus at increased risk for COVID-19 infection? Lancet Respir Med 8: e21.

19. Ming Y, Qiang L (2020) Involvement of spike protein, furin, and ACE2 in SARS-CoV-2-related cardiovascular complications. SN Compr Clin Med, 1-6.

20. Ejaz $H$, Alsrhani A, Zafar A, Javed $H$, Junaid $K$, et al. (2020) COVID-19 and comorbidities: Deleterious impact on infected patients. J Infect Public Health 13: 1833-1839.

21. Sanyaolu A, Okorie C, Marinkovic A, Patidar R, Younis K, et al. (2020) Comorbidity and its impact on patients with COVID-19. SN Compr Clin Med, 1-8.

22. Yang J, Zheng Y, Gou X, Pu K, Chen Z, et al. (2020) Prevalence of comorbidities and its effects in patients infected with SARS-CoV-2: A systematic review and metaanalysis. Int J Infect Dis 94: 91-95.

23. Orioli L, Hermans MP, Thissen JP, Maiter D, Vandeleene B, et al. (2020) COVID-19 in diabetic patients: Related risks and specifics of management. Ann Endocrinol (Paris) 81: 101-109.
24. Yan Y, Yang Y, Wang F, Ren H, Zhang S, et al. (2020) Clinical characteristics and outcomes of patients with severe covid-19 with diabetes. BMJ Open Diabetes Res Care 8: e001343.

25. Al-Sofiani ME, Ganji SS, Kalyani RR (2019) Body composition changes in diabetes and aging. J Diabetes Complications 33: 451-459.

26. Zhou F, Yu T, Du R, Fan G, Liu Y, et al. (2020) Clinical course and risk factors for mortality of adult inpatients with COVID-19 in Wuhan, China: A retrospective cohort study. Lancet 395: 1054-1062.

27. Kalyani RR, Golden SH, Cefalu WT (2017) Diabetes and aging: Unique considerations and goals of care. Diabetes Care 40: 440-443.

28. Bhatraju PK, Ghassemieh BJ, Nichols M, Kim R, Jerome $\mathrm{KR}$, et al. (2020) Covid-19 in critically ill patients in the Seattle region-case series. N Engl J Med 382: 2012-2022.

29. Grasselli G, Zangrillo A, Zanella A, Antonelli M, Cabrini L, et al. (2020) Baseline characteristics and outcomes of 1591 patients infected with SARS-CoV-2 admitted to ICUs of the Lombardy region, Italy. JAMA 323: 1574-1581.

30. Li Q, Guan X, Wu P, Wang X, Zhou L, et al. (2020) Early transmission dynamics in Wuhan, China, of novel coronavirus-infected pneumonia. N Engl J Med 382: 11991207.

31. Kumar A, Arora A, Sharma P, Anikhindi SA, Bansal N, et al. (2020) Is diabetes mellitus associated with mortality and severity of COVID-19? A meta-analysis. Diabetes Metab Syndr 14: 535-545.

32. de Almeida-Pititto B, Dualib PM, Zajdenverg L, Dantas JR, de Souza FD, et al. (2020) Severity and mortality of COVID 19 in patients with diabetes, hypertension and cardiovascular disease: A meta-analysis. Diabetol Metab Syndr 12: 75.

33. Pickup JC, Mattock MB, Chusney GD, Burt D (1997) NIDDM as a disease of the innate immune system: Association of acute-phase reactants and interleukin- 6 with metabolic syndrome X. Diabetologia 40: 1286-1292.

34. Knapp S (2013) Diabetes and infection: Is there a link?--A mini-review. Gerontology 59: 99-104.

35. Guo W, Li M, Dong Y, Zhou H, Zhang Z, et al. (2020) Diabetes is a risk factor for the progression and prognosis of COVID-19. Diabetes Metab Res Rev, e3319.

36. Zhu L, She ZG, Cheng X, Qin JJ, Zhang XJ, et al. (2020) Association of blood glucose control and outcomes in patients with COVID-19 and pre-existing type 2 diabetes. Cell Metab 31: 1068-1077.

37. Fuso L, Pitocco D, Antonelli-Incalzi R (2019) Diabetic lung, an underrated complication from restrictive functional pattern to pulmonary hypertension. Diabetes Metab Res Rev 35: e3159.

38. Acharya D, Lee K, Lee DS, Lee YS, Moon SS (2020) Mortality rate and predictors of mortality in hospitalized COVID-19 patients with diabetes. Healthcare (Basel) 8: 338.

39. Corona G, Pizzocaro A, Vena W, Rastrelli G, Semeraro F, et al. (2021) Diabetes is most important cause for mortality in COVID-19 hospitalized patients: Systematic review and meta-analysis. Rev Endocr Metab Disord 22: 275-296. 